\title{
Unexplained Increase in Death Rate during COVID19 Pandemic Mistakenly Attributed to Malaria
}

\author{
Alaaeldeen Mohammed Ahmed Abdeldafia, Ibrahim Abdelrhim Ali, Abrar Bakry Elmalik, and \\ Mohamed Eltayieb Elawad
}

\section{ABSTRACT}

Background: Since the emergence of COVID-19 pandemic, the world has faced many challenges. In Sudan, Gizera state has shown mysterious symptoms to the residents of a village and has been diagnosed with malaria.

Aim: The study was aimed to disprove the diagnosis of malaria, and to find another more convincing explanation that fits with the general features of the disease.

Method: A descriptive cross-sectional study was conducted using an electronic questionnaire designed by Google form.

Result: The respondents was 402 , the distribution of males and females was $51.7 \%$ and $48.3 \%$, respectively. $83 \%$ were suffered the symptoms in May and June. The most common symptoms were headache, fever, fatigue and joint pain, back pain, sore throat and anosmia, at $60.7 \%, 49.8 \%$, $47.3 \%, 33.3 \%, 37.3 \%$, and $23.3 \%$, respectively. $61.4 \%$ seeked health care, 99.9\% performed peripheral blood film for malaria and $83 \%$ were positive. $77 \%$ of those with positive result, $72 \%$ of those with negative results, $62 \%$ of those who did not seek health care suffered similar symptoms, mainly headache. Also, anosmia is $30 \%$, and $25 \%$ for those who have seeked and have not seeked health care, respectively. The recovery period was less than a week in $49 \%$ of those with positive results, $57 \%$ of those with negative results, and $63 \%$ of those who did not seek health care. It was more than two weeks in $9 \%, 12 \%$, and $4 \%$ in the positive, negative, and uncaring, respectively. $56.5 \%$ did not notice the mosquitoes in that period, $31 \%$ and $11.7 \%$ noticed that and did not know, respectively.

Conclusion: The most likely diagnosis of these mysterious symptoms is COVID-19.

Keywords: COVID-19, Malaria, Gizera state, Sudan.

\section{INTRODUCTION}

Since the World Health Organization (WHO) declared COVID-19 to be pandemic, the world has been undergoing changes in all aspects of life in general, and in the medical space in particular. Countries have had difficulties in absorbing the number of infected people, providing protective equipment for medical personnel, and ensuring stable health conditions for people [1].

This pandemic has proved its importance and well-treated in particular in several ways, the most prominent of which is that the number of deaths was estimated globally at 139.378. The total confirmed new cases were 2,074,529, according to the WHO report in April 2020 [2].

This reality obliges the health sector to face many difficult challenges, which are represented in the collapse of health systems or the fact that they are overloaded with their capacity, the number of deaths per day is soaring. Design
Published Online: November 14, 2020

ISSN: 2593-8339

DOI: $10.24018 /$ ejmed.2020.2.6.545
Alaaeldeen Mohammed Ahmed Abdeldafia
King Abdulaziz Medical City, Jeddah, Saudi Arabia.
Ibrahim Abdelrhim Ali*
Department of Physiology, Faculty of Medicine, The National Ribat University, Khartoum, Sudan.
(e-mail: hemamedicine ${ }^{\circledR}$ gmail.com)
Abrar Bakry Elmalik
Faculty of Medicine, Omdurman Islamic University, Khartoum, Sudan.
Mohamed Eltayieb Elawad
Department of Physiology, Faculty of Medicine, The National Ribat University, Khartoum, Sudan.

*Corresponding Author surveillance systems and surveys to collect the required data about the community and provide diagnostic tools to identify positive cases and isolate them to reduce infection spread [3].

Also, countries have made many attempts that are expected to reduce contagion, such as: travel restrictions, closures of educational institutions, and suspension of activities of all kinds [4], [5].

The diagnosis of the novel Corona virus disease is one of these challenges, as difficulty in recognizing the disease is shown in unusual symptoms that are often similar to symptoms of other diseases. More ominously, these diseases are often known and pervasive in the region. In tropical regions, a COVID-19 case was recorded in Thailand, where the disease was a form of dengue fever, and the latter is a common disease there. Not only is this, but the number of platelets was low, which led the doctor to diagnose him with dengue fever in the first presentation [6]. 
The dilemma that is created by this symmetry can have serious consequences, the worst of which is the rise of the mortality curve. This means the loss of human force and this is negatively reflected in the economy already affected by several ways [7]. In addition, a misdiagnosis may give a patient a good sensation in dealing with his or her surroundings, which can be translated into the virus transmission chain reinforcement and expansion of the new cases space.

The situation can worsen with COVID-19 associated diseases, where the majority of patients are found to have hypertension, diabetes, heart disease, respiratory diseases, and cancerous tumors which all can contribute to an increased COVID-19 severity, poor outcomes, and a 5\% increase in mortality rate [8]-[10].

In Sudan, specifically in Gezira state during the Corona pandemic, people have suffered mysterious symptoms similar to malaria, but they differ from it in many other aspects and the number of deaths has risen dramatically. There was no convincing explanation for what happened, except their diagnosis of malaria. Even this diagnosis lacked the overwhelming evidence to support it. In this study, we aim to refute that diagnosis and find another compelling explanation for that strange phenomenon.

By this research, we are seeking to uncover the truth by which we can illustrate the image of actual new cases, which means designing preventive plans that target the affected areas in a focused manner. And providing the right health care to members of that population. All of this positively affects high mortality rate and helps us to manage well and control infection.

\section{METHODS}

A descriptive cross-sectional study was conducted in Gezira state in Sudan from April to June 2020 during COVID 19 pandemic infection using an electronic questionnaire designed by Google form and covered personal, social and medical history. The symptoms related to Malaria and COVID 19 infection have been illustrated in the questionnaire.

There were 402 respondents living in the targeted village in Gezira state.

Data is analyzed using SPSS version 25.0 and the variables studied are described by percentages. P-value is less than 0.05 was considered statistically significant.

\section{RESULTS}

The total number of people that enrolled in this study were 402 person, $51.7 \%$ were female and $48.3 \%$ were male, $324(83 \%)$ had complain in recent two month, the complains and symptoms are different as shown in Table 1.

The most common symptoms are headache $(60.7 \%)$, fever $(49.8 \%)$, fatigability and joint pain $(47.3 \%)$, back pain (33.3\%), sore throat (37.3\%) anosmia (23.3). When we asked about if there is any one of the family or friends experienced one these symptoms, $70.1 \%$ answered yes and the remaining (29.9) said no.
TABLE 1 SYMPTOMS AND COMPLAINTS OF STUDY POPULATION

\begin{tabular}{cc}
\hline Symptoms & The percentage \\
\hline Headache & $60.7 \%$ \\
Fever & $49.8 \%$ \\
Fatigability and joints pain & $47.3 \%$ \\
Sore throat & $37.3 \%$ \\
Back pain & $33.3 \%$ \\
Anosmia & $23.9 \%$ \\
Sweating & $21.9 \%$ \\
Ageusia & $15.7 \%$ \\
Productive cough & $13.7 \%$ \\
Dry cough & $13.7 \%$ \\
Shortness of breath & $10.4 \%$ \\
Night marries and hallucination & $9.5 \%$ \\
Diarrhea & $8.5 \%$ \\
Skin changes & $2.7 \%$ \\
\hline
\end{tabular}

Most of the people went to health center to seek advises (61.4\%), and the remaining (38.6) did not seek any medical service. From those who went to health centers almost all did blood test for malaria parasite (198 equal 99.9\%) the result was positive to malaria in $83 \%$ of people tested, and $17 \%$ were negative to malaria.

92 persons had insomnia. Most of them (54.4\%) were tested positive for malaria, $11 \%$ were tested negative and the remaining $34 \%$ were not seeking medical advises.

People who tested positive or negative to malaria and those did not go to health center were experience almost same symptoms and complaints, as shown in Fig. 1, for example headache as a symptoms was the main symptoms of all these groups with near equal percentage $(77 \%$ for one whose tested positive, $72 \%$ for tested negative and $62 \%$ for not seeking medical advice), another example is anosmia which was experienced in $30 \%$ in whose tested positive and negative and $25 \%$ for people that no seeking medical advises.

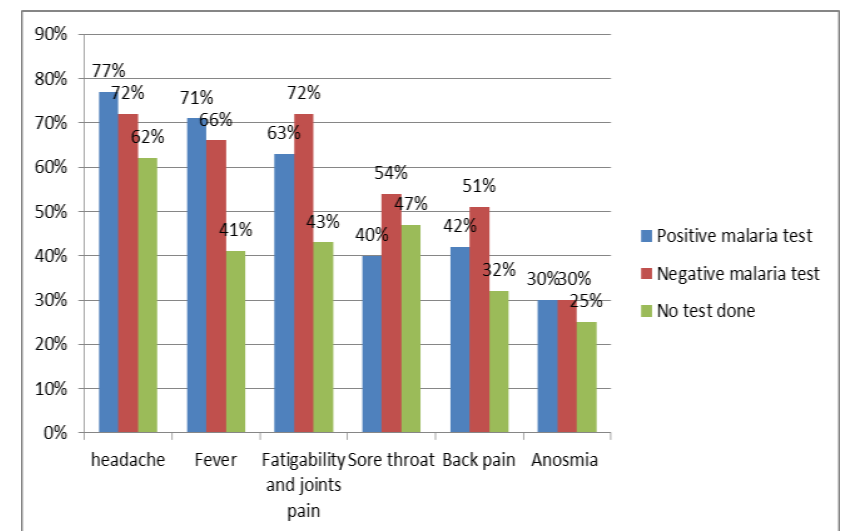

Fig. 1. Comparison of the most common symptoms of a group that has been found to be positive for malaria, negative for malaria, and those who did not test.

The recovery time of the symptoms and complaints were almost not significant different between the groups, it was less than one week in $49 \%$ for who tested positive for malaria, $57 \%$ for who tested negative for malaria, and $63 \%$ for group that did not seek medical advice. Recovery time was more than one week and less than two week for $25 \%$, $15 \%$ and $19 \%$ for tested positive, tested negative and for people that did not seek medical advice. 9\% of tested positive group said the symptoms took more than two weeks 
to disappear, $12 \%$ and $4 \%$ for tested negative group and group did not seek medical advises, respectively.

Most of people $(56.5 \%)$ did not notice increase in the number of mosquitos, $31 \%$ noticed increase in the mosquitos, the remaining $11.7 \%$ they said they did not know.

\section{DISCUSSION}

This study was done in a village in Gizera state, in two month (May and June 2020) there were 33 persons died, almost all of them are elderly with known chronic illness, most of those people were die at home with primary diagnoses was malaria, one of them did seek medical advised and diagnosed as COVID 19 infection, the others were treated at home by local health centers. There is rumor in the local community that all of people were died as result of this new type of malaria as locally called Eritrean malaria (they mean malaria vivax).

- From this study result we found the increase deaths in last two months was not due to malaria and we believe it was due to COVID 19 infection because of the followings points The percentage of positive malaria cases from those get tested was too high $83 \%$ and not acceptable when we compared it with documented percentage of positive cases at governmental health center which located at same area, this health center is managed by ministry of health which has regular check on this center to insure quality of malaria testing and take the official number of malaria cases per month, also the laboratory technicians that work in the governmental health center are well trained for malaria testing and they have very long experience in this field. In reports of ministry of health, the percentage of positive malaria cases from tested patients was 5 to $9 \%$ at the same months in last year and it did not change since January 2020 as shown in Fig. 2. So, when compare it with our result in this study we will find that there were many cases was tested positive to malaria and they were not, this happened because of most of people in the study area seek medical advice from private health centers which has lack of supervision from ministry of health and no one applied quality controlled. After we brought this concern to the ministry of health and local health authority, they collected 20 slides from each private health center from the cases which they said were tested positive for malaria, the ministry of health after checking the sample,they refused all the samples from the three private health centers due to inappropriate sample taking and staining even they did not check it under microscope and they sent team to train the working laboratory technicians to how take and perform malaria test.

- Increase the malaria cases related to increase in mosquito's number, but most of people in the study they did not noted any increase in the number of the mosquitoes and just $31 \%$ noted this.

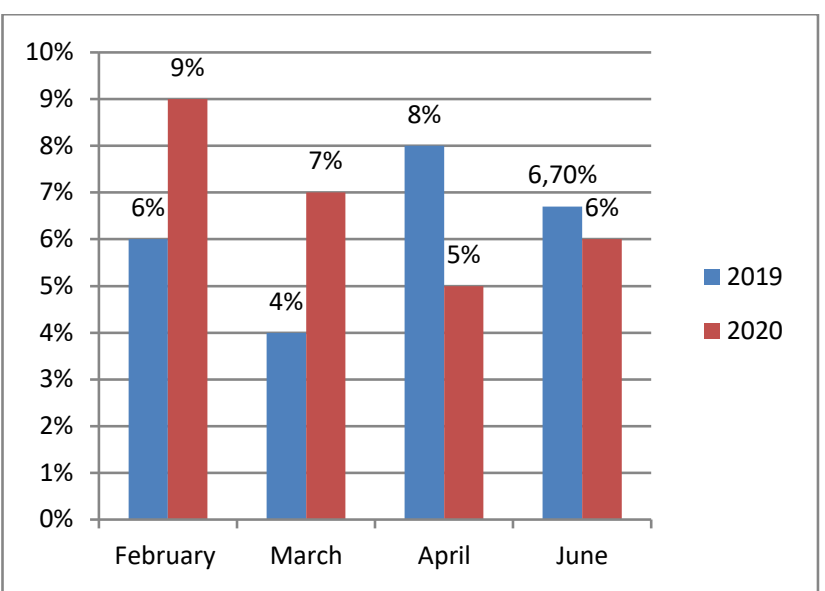

Fig. 2. The percentage of positive samples for malaria from the tested samples in months from February to June 2020 and in 2019.

- People that tested positive to malaria and received treatment and those tested negative and those did no go to any health centers were almost took same time to recover from their symptoms as shown in fig most of people their symptoms disappear in one week as this exactly the time needed to corona virus symptoms release in most patients.

- People who tested positive for malaria and those tested negative and those did not go to any health center were almost experienced same symptoms and take almost same period to recover from their symptoms, it did not matter if they tested positive for malaria of negative or even not went to health center, so this mean all this groups had same disease which can be relived spontaneously without any management, this criteria of disease is like most of the COVID 19 patients which are experienced symptoms that relieves spontaneously and they do not need admission.

- There is 92 persons who were complaining of anosmia more than half diagnosed as malaria and we know that anosmia is not a common symptoms of malaria and in many studies they describes anosmia is one of symptoms of COVID 19 infection.

- We brought our concern to local health authorities which they came to the area and try to take some of suspected sample, no one from the population agree to take the sample and after week they convinced two young guys to do corona test, one of them was positive and the other was negative, more ever one of the deaths happen in hospital and they tool sample and it was positive to corona, so the corona virus is spreading through entire population but no one did the investigation required.

- There are about $70.1 \%$ of the sample say yes, if one of their family members or friends has the same symptoms. From this point, we inferred that these symptoms are caused by a contagious disease that is likely to be COVID-19 not malaria, which is not contagious.

- Almost all of the dead cases were elderly, meaning that age is a risk factor for the disease. Advanced age is a factor associated with increased COVID-19 severity that can lead to death, unlike malaria.

- Almost all of the deaths were chronic disease patients, 
which show that chronic illnesses are a risk factor for this disease and have the ability to increase the severity of COVID-19, which can lead to death, unlike malaria.

- The majority of deaths were at home from those who were previously diagnosed with malaria, and one of them had seeking medical advice again and had been diagnosed with COVID-19. This individual suffered from the same symptoms as others and lived with them in the same community, so we can generalize the positive result of HIV infection throughout the population.

\section{CONCLUSION}

In this paper, we have refuted the malaria diagnosis of the unexplained features that have emerged in the village population in several points, and have suggested that it could be a COVID-19 and have provided convincing evidences of this suggestion through the results obtained from the questionnaire.

It is very likely that the COVID-19 and other diseases in a specific geographic area similar in clinical manifestations are confused, as in that village in gizera state where high incidence of malaria and limited diagnostic means are available.

We recommend that the competent authorities provide sophisticated equipment and well-trained medical personnel in remote villages to detect the corona virus at early stages in order to prevent the disease from spreading in a way that is difficult to control.

\section{DECLARATIONS}

Consent to participate:

All participants signed a written informed consent form. Availability of data and material:

The datasets used and/or analyzed during the current study are available from the corresponding author on reasonable request.

Competing interests:

The authors declare that they have no competing interests. Funding: None.

\section{REFERENCES}

[1] Sohrabi C, Alsafi Z, O’Neill N, Khan M, Kerwan A, Al-Jabir A, Iosifidis C, Agha R. World Health Organization declares global emergency: A review of the 2019 novel coronavirus (COVID-19). International Journal of Surgery. 2020 Feb 26.

[2] World Health Organization. Coronavirus disease 2019 (COVID-19): situation report, 88 .

[3] Onder G, Rezza G, Brusaferro S. Case-fatality rate and characteristics of patients dying in relation to COVID-19 in Italy. Jama. 2020 May 12;323(18):1775-6.

[4] Kraemer MU, Yang CH, Gutierrez B, Wu CH, Klein B, Pigott DM, Du Plessis L, Faria NR, Li R, Hanage WP, Brownstein JS. The effect of human mobility and control measures on the COVID-19 epidemic in China. Science. 2020 May 1;368(6490):493-7.

[5] Flaxman S, Mishra S, Gandy A, Unwin HJ, Mellan TA, Coupland H, Whittaker C, Zhu H, Berah T, Eaton JW, Monod M. Estimating the effects of non-pharmaceutical interventions on COVID-19 in Europe. Nature. 2020 Aug;584(7820):257-61.

[6] Joob B, Wiwanitkit V. COVID-19 can present with a rash and be mistaken for Dengue. Journal of the American Academy of Dermatology. 2020 May;82(5): e177.
[7] Nicola M, Alsafi Z, Sohrabi C, Kerwan A, Al-Jabir A, Iosifidis C, Agha M, Agha R. The socio-economic implications of the coronavirus pandemic (COVID-19): A review. International journal of surgery (London, England). 2020 Jun; 78:185.

[8] Guan WJ, Liang WH, Zhao Y, Liang HR, Chen ZS, Li YM, Liu XQ, Chen RC, Tang CL, Wang T, Ou CQ. Comorbidity and its impact on 1590 patients with Covid-19 in China: A Nationwide Analysis. European Respiratory Journal. 2020 May 1;55(5).

[9] Yang J, Zheng Y, Gou X, Pu K, Chen Z, Guo Q, Ji R, Wang H, Wang $\mathrm{Y}$, Zhou Y. Prevalence of comorbidities in the novel Wuhan coronavirus (COVID-19) infection: a systematic review and metaanalysis. International journal of infectious diseases. 2020 Mar 12.

[10] Li LQ, Huang T, Wang YQ, Wang ZP, Liang Y, Huang TB, Zhang HY, Sun W, Wang Y. COVID-19 patients' clinical characteristics, discharge rate, and fatality rate of meta-analysis. Journal of medical virology. 2020 Jun;92(6):577-83. 